\title{
TIME-DEPENDENT MODEL CALCULATIONS FOR THE MOLECULAR HYDROGEN ION IN A STRONG ULTRA-SHORT LASER PULSE
}

\author{
G. Ver Steeg and K. Bartschat
}

Department of Physics and Astronomy, Drake University, Des Moines, IA 50311, USA

We have revisited the problem of a hydrogen molecular ion in the presence of a short intense laser field. Our approach for time-propagation the initial state subject to the Schrödinger equation is similar to that presented by Kulander et al. [1]. A common simplification to the full problem is the reduction to one dimension with two degrees of freedom, one for the nuclear separation $(R)$ and one for the electronic motion along the internuclear $(z)$ axis, with the electric field also along this axis. The goals of the present project are: 1) to apply our method, developed for $\mathrm{e}-\mathrm{H}$ collisional excitation and ionization [2], to an explicitly time-dependent problem; 2) to check different time-propagation schemes; 3 ) to visualize the results; 4) to extract the physics by projection techniques; and 5) to try avoiding masking potentials that reduce the norm of the wavefunction.

Going beyond the Born-Oppenheimer approximation, we solve the time-dependent Schrödinger equation

$$
\begin{array}{r}
i \frac{\partial \Psi(R, z, t)}{\partial t}=\left[-\frac{1}{2 \mu} \frac{\partial^{2}}{\partial R^{2}}-\frac{1}{2} \frac{\partial^{2}}{\partial z^{2}}+\frac{1}{\sqrt{R^{2}+q_{n}}}\right. \\
-\frac{1}{\sqrt{(z-R / 2)^{2}+q_{e}}}-\frac{1}{\sqrt{(z+R / 2)^{2}+q_{e}}} \\
\left.+z f(t) \mathcal{E}_{0} \sin (\omega t)\right] \Psi(R, z, t) \quad(1)
\end{array}
$$

by time-propagating the initial state

$$
\Psi(R, z, t=0)=F_{v}^{1 \sigma_{\mathrm{g}}}(R) G_{R}^{1 \sigma_{\mathrm{g}}}(z),
$$

where $G_{R}^{1 \sigma_{\mathrm{g}}}(z)$ is the electronic ground state for fixed $R$ and $F_{v}^{1 \sigma_{\mathrm{g}}}(R)$ is the vibrational state $v$.
The screening parameters $q_{n}=0.03$ and $q_{e}=1.0$ are used to smooth out the Coulomb singularity. The term $z f(t) \mathcal{E}_{0} \sin (\omega t)$ represents the effect of the electric field with amplitude $\mathcal{E}_{0}$ and angular frequency $\omega$. Finally, $f(t)$ is a smooth turn-on/turn-off function for the field.

We have used intensities of $1-2 \times 10^{18} \mathrm{~W} / \mathrm{m}^{2}$, turn-on/turn-off times of $2-4$ periods, and ontimes up to about 20 periods. We also follow the system for several periods after the pulse is over. Typical observables to calculate are $\langle R(t)\rangle$ and $<z(t)>$. However, $\langle R(t)>$ has limited meaning since it includes vibration, dissociation, and Coulomb explosion; the same is true for $\langle z(t)\rangle$. Looking at the complete nuclear and electronic distributions reveals significantly more information.

We therefore created movies to watch for bond-stretching and bond-healing, vibrational excitation, dissociation (electron stays with one of the protons) and ionization (electron leaves the system and protons undergo "Coulomb explosion"). Links to these movies can be found at http://bartschat.drake.edu/. We are currently working on our projection techniques to find, for example, the probability of exciting a particular vibrational and/or electronic state.

This work was supported by the United States National Science Foundation through an REU supplement to grant PHY-0088917.

\section{References}

[1] K.C. Kulander, F.H. Mies and K.J. Schafer, Phys. Rev. A 53 (1996) 2562.

[2] K. Bartschat, S. Riordan and G. Ver Steeg, Phys. Rev. A 65 (2002) 060701(R). 